Published in final edited form as:

Curr Opin Struct Biol. 2009 August ; 19(4): 440-441. doi:10.1016/j.sbi.2009.07.010.

\title{
Engineering and Design:
}

\section{Editorial overview}

\author{
Brian Kuhlman and \\ University of North Carolina, Department of Biochemistry and Biophysics, 120 Mason Farm Rd, \\ Chapel Hill, NC 27599-7260, USA, bkuhlman@email.unc.edu

\section{William F DeGrado} \\ University of Pennsylvania, School of Medicine, Department of Biochemistry \& Biophysics, \\ Philadelphia, PA 19104-6059, USA, wdegrado@mail.med.upenn.edu
}

In this section we have assembled a set of reviews that focus on the design of proteins and peptides with new molecular recognition capabilities. These include proteins that promote crystallization of target proteins, peptides useful for creating nanoelectronic circuits, peptide therapeutics, and switchable enzymes that respond to novel molecular inputs.

Allosteric modulation of enzyme activity is typically rapid and reversible and is used to create positive and negative feedback loops in cellular signaling networks. Marc Ostermeier reviews progress in the engineering of switchable enzymes that respond to new signaling inputs. One common strategy is to fuse a catalytic domain with a binding domain that changes conformation when bound to a target ligand. The conformational change can be used to disrupt or assemble the active site as well as relieve steric inhibition of activity. Circular permutation of the host protein allows for a variety of fusion sites, for which the best sites can be identified computationally or with high-throughput screening. There is considerable interest in the design of new switches because they can be used to probe interaction dependencies and kinetics in signaling cascades.

Shohei Koide describes recent progress in engineering crystallization chaperones. These are proteins that bind to specified target molecules and promote crystal formation by reducing conformational heterogeneity of the target and providing extra surface area for crystal contact formation. They can also increase or decrease the solubility of the target protein as needed. Fragments of monoclonal antibodies were among the first proteins used as crystallization chaperones and have been used to help solve structures of several important membrane proteins. However, monoclonal antibodies are slow to create and their production at a milligram scale is expensive. Koide describes several newer strategies that do not require animal immunization, but rather use molecular display technologies to identify proteins that bind tightly to the crystallization target. One advantage of this approach is that it can be used with a variety of protein scaffolds, including single chain antibodies, ankyrin repeat proteins, and fibronectin domains. This approach has been used to crystallize the fulllength form of the potassium channel KcsA as well as wild-type Polo-like kinase 1. One important drawback of the chaperone approach is that it requires the production of a new chaperone for each target, for this reason, this technology will in general be reserved for high impact targets.

Protein design tests our understanding of protein structure and stability. This is particularly true of computer-based studies that use physical models of protein energetics to predict amino acid sequences that will form predetermined structures, complexes or binding sites. John Karanicolas and Brian Kuhlman review recent progress in computational design of protein-protein interactions. They focus on strategies developed for enhancing the affinity of naturally occurring interactions and redesigning protein binding specificities. Particularly 
exciting is progress in the use of explicit negative design to create sequences that bind to specific targets within large protein families.

There is considerable interest in designing proteins that adopt specific conformations when embedded in a membrane. Critical to such efforts is a good understanding of the factors that determine the forces and sequence features that dictate the insertion of transmembrane helices into membranes. Erwin London and Khurshida Shahidullah review studies that probe the relationship between sequence and helix topography in membranes. These include the effects of overall hydrophobicity, helix length, polar residue placement, and the role of flanker sequences. They demonstrate that there is a remarkable similarity between the conclusions from experiments involving the translocon-mediated insertion of model peptides and biophysical studies of fully synthetic peptides in model membranes. These findings suggest that the interaction of potential TM segments with the lipid bilayer during their residence in the translocon, or possibly that the environment experienced by a helix in the pore of the translocon is similar to that of a phospholipid bilayer. Irrespective of the precise mechanism, this paper provides a lucid overview of the features that must be considered in designing and predicting transmembrane helices.

Fred Naider and Jacob Anglister describe how structural biology studies have directed the design of peptides that bind to the HIV-1 envelope protein gp41 and inhibit fusion of the virus to target cells. One of these peptides is currently used in the clinic in cases where patients have developed resistance to more standard cocktails of protease and transcriptase inhibitors. This paper provides a particularly interesting analysis of the role of structural, thermodynamic, and kinetic studies in the design and analysis of inhibitors of gp41, and also in devising strategies to combat the growing problem of resistance to this class of pharmaceutical agents.

In addition to being useful as regulators of biological systems, peptides can be engineered to form novel materials. Robert Fairman and Brian Pepe-Mooney examine recent progress toward creating nanocircuits based on peptides. They describe new strategies for depositing peptides on surfaces and methods for attaching cofactors that can be used to generate new electronic properties.

\section{Biographies}

Brian Kuhlman received his BA from Rice University and his PhD from SUNY Stony Brook working with Daniel Raleigh. As a postdoctoral fellow in David Baker's laboratory at the University of Washington he developed and tested software for protein design. His laboratory at the University of North Carolina is focused on de novo protein design, protein interface design, and the design of protein switches include the optimization of photoactivatable proteins that can be used in living cells to reversibly activate signaling pathways, and the creation of altered specificity binders for probing dependencies in signaling networks.

Bill DeGrado received his $\mathrm{PhD}$ from the University of Chicago, and worked at DuPont and DuPont Merck for 15 years before joining the University of Pennsylvania. His work focuses on the analysis and design of proteins and protein mimics. 